\title{
Un estudio multidisciplinario interinstitucional de necesidades sociocomunicativas lingüísticas: hacia una propuesta curricular para la ingeniería agroindustrial, 2014, CURVA-ELCE UNAH
}

José Alexis Espino, Jean Noel Cooman, Oscar Arlyn Gálvez ${ }^{1}$

\section{RESUMEN}

Con el interés de responder a la demanda de un diseño curricular de parte de la Carrera de Ingeniería en Agroindustria del Centro Universitario Regional del Valle de Aguan (CURVA) en la ciudad de Olanchito, se inició, dentro de la Escuela de Lenguas y Culturas Extranjeras, a finales del año 2014, la realización de un estudio científico con un enfoque cuanti-cualitativo, que recogió datos de 4 poblaciones idóneas, con miras a la elaboración de una propuesta curricular para el programa de inglés por objetivos específicos para dicha carrera en este centro regional de la Universidad Nacional Autónoma de Honduras (UNAH).

Este proyecto de investigación tuvo como objetivo, en primer lugar, diagnosticar y determinar a través de 5 instrumentos de recolección de información, las exigencias de formación en la lengua inglesa y, después del análisis de los datos obtenidos, constituir y validar técnicamente un programa nuevo y más intensivo que responda a las necesidades objetivas-comunicativas, necesidades subjetivas y necesidades interculturales específicas de los estudiantes y egresados de Ingeniería en Agroindustria; de tal manera que los lleve eventualmente a adquirir mejores competencias comunicativas-lingüísticas para un óptimo aprovechamiento académico y profesional en esa área en concreto.

El presente estudio consiguió determinar el nivel actual e insuficiente de la lengua inglesa de la población de estudiantes; la demanda, motivación y hábitos de estudio actuales de los mismos para el aprendizaje de la lengua; al igual que los contenidos,

\footnotetext{
${ }^{1}$ Beneficiarios de una beca básica, profesores investigadores, Escuela de Lenguas Extranjeras Facultad de Humanidades y Artes, UNAH: alexisespino2009@yahoo.com, jnoecooman@yahoo.com
} 
competencias sociolingüísticas, estrategias de enseñanza y metodología de evaluación deseables. Es decir, se determinó lo que estos estudiantes deben de leer, escuchar, redactar y hablar en inglés de manera más orientada a sus necesidades profesionales. En conclusión, se respondió a la interrogante: “¿Quién tiene necesidad de qué lengua para efectuar qué operación de acuerdo a qué interlocutor dentro de qué circunstancia y para qué objetivo, a propósito de qué objeto, con ayuda de qué medios y de qué formas lingüísticas?" (Cabré, 2010). Estos componentes dieron forma a la propuesta curricular formulada.

Consecuentemente, la gestión de esta novedosa reforma curricular pretende impulsar el desarrollo socioeconómico de las familias de la comunidad y, paralelamente, dar respuesta a la demanda de contratación de profesionales capacitados en las cuantiosas empresas nacionales e internacionales de la zona del valle del Aguán, dedicadas al rubro de la agricultura y la industria.

Palabras claves: ESP (english for specific purposes), necesidades objetivas-comunicativas, interculturales, competencias lingüísticas.

\section{ABSTRACT}

In the interest of responding to the demand of the Career of Engineering in Agribusiness of the Centro Universitario Regional del Valle de Aguan (CURVA) in the city of Olanchito, Honduras, a scientific study was conducted on a quantitative and qualititive based approach, at the Foreign Languages Department of the UNAH, late 2014; such research collected data from 4 appropriately selected populations, with the aim of designing and developing a curriculum for the English for Specific Purposes (ESP) program of this regional center of the National Autonomous University of Honduras (UNAH).

This research project was aimed, first, to diagnosing and determining, through 5 instruments for data collection, the training needs in English (the needs of education in English) and then, after the analysis of these data, to establishing and technically validating a more intensive program that will respond to profession-orientated communicative and linguistic needs, subjective needs and intercultural needs of students and graduates of the Engineering in Agribusiness. The new program will eventually lead to acquire better language and communicative skills for optimal academic and professional achievement in this concrete area. 
In short, this study determined the students' insufficient level of English, the demand, motivation and actual study habits to learning the language, also the needed content, sociolinguistic competence, teaching strategies and assessment methodology. Moreover, the research determined how these students should read, listen, write and speak in English in a more profession-orientated way. In conclusion, the following question was answered: "Who needs what language, which operation to perform, according to what party, in what circumstances and for what purpose, with regard to which object, using what means and in what language forms ? ", Cabré (2010). These components shaped the curriculum proposal that was formulated.

Consequently, the management of this new curriculum reform attempts to promote the socioeconomic development of the families in the community, and to respond to the demand of hiring skilled professionals because of the huge number of national and international companies in the area of Valle del Aguan, engaged in the field of agriculture and industry.

Keywords: ESP (english for specific purposes), profession-orientated communicative and linguistic needs and intercultural skills. 


\section{INTRODUCCIÓN}

En el contexto de creciente globalización y en el mundo de conocimientos actuales, el aprendizaje de las lenguas extranjeras se ha convertido en una necesidad ineludible en el ámbito académico escolar, cada día más internacional, y en el profesional. En este sentido, el compromiso social de la Universidad Nacional Autónoma de Honduras (UNAH) manda a emprender proyectos investigativos que ayuden a resolver problemas institucionales, gremiales, comunales y nacionales a partir de planteamientos de reformas educativas novedosas.

En consecuencia, este artículo tiene como finalidad mostrar como la Escuela de Lenguas y Culturas Extranjeras (ELCE) de la ciudad universitaria de la UNAH, ha emprendido responsablemente, a petición de varias facultades, investigaciones diagnósticas con miras hacia el diseño curricular para la enseñanza del Inglés y otras lenguas, mediante objetivos específicos, lo que se define como: "Tomar en consideración las necesidades e intereses de los alumnos en relación con la lengua extranjera para el logro de su éxito y efectividad de su aprendizaje" (Mangiane, 2007), tal es el caso de la Carrera de Comercio Internacional, en la cual en el año 2013 ya se diseñó y a partir del año 2014 se está desarrollando dicho programa en la modalidad de diplomado de lengua especializada; de la misma manera, otras unidades y facultades como Antropología, Ingeniería, Ciencias Médicas; una facultad del Centro Universitario Regional del Litoral Atlántico (CURLA), el Instituto Técnico Superior de Tela (ITST-UNAH), así como la Carrera de Ingeniería en Agroindustria del Centro Universitario Regional del Valle de Aguán (CURVA), entre otras, las que también han solicitado la creación de propuestas curriculares que lleven a satisfacer las necesidades de formación de competencias en la lengua inglesa en estas áreas específicas.

En lo que respecta a la Carrera de Agroindustria del CURVA, la formación en inglés general que reciben actualmente los estudiantes en los cursos de lengua extranjera Inglés 101, 102 y 103, está caracterizada, como lo señala Mangiante (2007), por: "Un sentido comunicativo muy general de la lengua, mas no un sentido particular del ámbito"; en este caso de la ciencia de la agroindustria. Además: "La enseñanza debe estar concebida en función del estudiante y es hacia él que deben orientarse las diferentes acciones a realizar" (Porcher, 2004). En este sentido, lo ideal y urgente es brindarle al estudiante y profesional egresado los conocimientos teóricos- prácticos y los aspectos sociolingüísticos: "Necesarios para que desarrollen competencias de comprensión auditiva y de lectura, así como de expresión escrita y oral en inglés para una óptima ventaja académica y socioeconómica propia de este campo disciplinar" 
(Mangiante y Parpette, 2004).

Sin embargo, la sección de inglés del CURVA ha carecido hasta el momento de un programa de aprendizaje de la lengua inglesa que permita eficaz y eficientemente a este grupo de estudiantes y profesionales egresados desarrollar destrezas y habilidades auditivas, de lectura, escritura y producción oral, propias y oportunas en su campo de conocimiento y su ámbito sociocultural.

En cuanto a la diferencia entre una formación en lenguas por objetivos específicos y una formación en lenguas de especialidad, Cabré $(2010,5-7)$ explica que en una formación de especialidad: "La adquisición de los recursos significa que el estudiante ya ha adquirido todo lo que necesita saber sobre su especialidad. Debemos suponer que el estudiante sí habrá adquirido la terminología propia de la materia, ya que difícilmente los especialistas habrían podido explicarse eficientemente sin recurrir a los conceptos propios del ámbito". Asimismo, en una formación por objetivos específicos, se toma en cuenta que: "...Su conveniencia comunicativa y los tipos de texto (oral o escrito) que deberán producir en situaciones de formalidad profesional, las estrategias representativas, expresivas y comunicativas necesarias para manejarse adecuadamente y eficazmente" (Cabré 2010, 5-7).

Igualmente, se debe dar una gran importancia significativa al aspecto cultural en el aprendizaje de una lengua por objetivos específicos, ya que no solamente se trata de aprender y hablar la lengua, sino también de saber cuándo y cómo usarla de una manera culturalmente aceptable. En este sentido: “...Es necesario desarrollar una cierta sensibilidad en los estudiantes, hacia los prejuicios sociales y culturales que conllevan determinados actos de habla" (Vez 2001,172-183).

También, el Marco Europeo Común de Referencias para las Lenguas (2005) establece como parte indispensable e insoslayable de las habilidades y destrezas a desarrollar en el estudiante, el componente de destrezas y habilidades pluri e interculturales. Entonces, el estudiante necesita desarrollar la sensibilidad cultural y capacidad de establecer contacto y relacionar entre sí su cultura materna y las culturas extranjeras que adquiera, en el momento de aprender y hablar sus nuevas lenguas extranjeras. Por esta razón, el problema investigativo que se planteó es el siguiente: ¿Cuáles son las necesidades subjetivas o de motivación, las necesidades objetivas-comunicativas lingüísticas, las necesidades de aprendizaje y las necesidades socioprofesionales 0 y las necesidades interculturales que requieren satisfacer los estudiantes y profesionales egresados de la Carrera de Ingeniería en Agroindustria, 2014-2015 del CURVA y que respondan a los objetivos específicos de su disciplina? 
En conclusión, este artículo logra destacar, por una parte, el carácter eminentemente pertinente y urgente; y por otra parte, el alcance multidisciplinar del estudio realizado, en el cual se combinan los conocimientos (habilidades), el "know how" específico del campo disciplinar de la agroindustria (en el valle del Aguán) y el "expertise", competencias específicas de la disciplina de la didáctica de lenguas y culturas. Más concretamente en el ámbito de las formaciones en lenguas por objetivos específicos o por objetivos universitarios, que cobran cada día más importancia y relevancia.

Esta dimensión multidisciplinar se está implementando además en un contexto interinstitucional, en el cual se ha conformado un equipo de docentes especialistas del inglés (de varios idiomas), de la ELCE y de la sección de inglés del CURVA. Finalmente, conviene destacar la presencia de la vinculación social en este estudio al involucrar a las empresas nacionales e internacionales de la región (del valle del Aguán) y dar respuestas a las exigencias acerca del perfil profesional deseado.

\section{OBJETIVOS DEL ESTUDIO}

Los estudiantes y egresados de la Carrera de Ingeniería en Agroindustria de la UNAH- CURVA se enfrentan, constantemente, a la necesidad de, entre muchas otras, leer y redactar textos en inglés, comprender y realizar exposiciones y negociaciones, asistir a investigaciones de campo y comprender conferencias virtuales y presenciales en el idioma inglés como parte de sus cursos curriculares; además, prácticas profesionales, desempeño laboral, así como parte de trabajos de investigación. Específicamente, los egresados de esta Carrera enfrentan retos frecuentes en su desempeño profesional en esta región hondureña con una importante presencia de empresas nacionales e internacionales del rubro de la agroindustria.

Es así que este estudio se propuso alcanzar el siguiente objetivo general: diagnosticar alrededor de las necesidades de formación en la lengua inglesa de los estudiantes y egresados de la Carrera de Ingeniería en Agroindustria del CURVA- UNAH, con el fin de diseñar y luego validar una propuesta curricular de enseñanza-aprendizaje de la lengua inglesa que responda a las necesidades personales, lingüísticas y socioprofesionales específicas de este público y su disciplina. 


\section{METODOLOGÍA}

La metodología utilizada para la realización de este estudio fue de tipo mixto: cuantitativo- cualitativo. Se recurrió a un diseño de tipo transversal exploratorio-descriptivo no experimental. Descriptivo, porque se buscó especificar características y perfiles de individuos, los cuales fueron sometidos a un análisis de sus necesidades subjetivas, objetivas-lingüísticas, de aprendizaje y socioculturales dentro del ámbito profesional que poseen los estudiantes.

En calidad de población informante participaron 44 alumnos, lo que representa el 100 $\%$ de los estudiantes matriculados actualmente en la Carrera de Ingeniería en Agroindustria del CURVA.

Del mismo modo, se consideró el $100 \%$, o sea, la totalidad de docentes y autoridades: 12, que laboran actualmente en la Carrera. También se identificó una muestra a conveniencia de 15 egresados que laboran en la zona, de una totalidad de 28; y otra muestra a conveniencia de 5 compañías de la región que emplean a los egresados de dicha Carrera.

El equipo investigador viajó y se alojó con su equipamiento en la ciudad de Olanchito y en las instalaciones del CURVA para sostener reuniones de trabajo y la aplicación de los siguientes instrumentos y técnicas para realizar el proceso de recolección de datos:

1. Prueba de medición inicial: midió el nivel de lengua actual y real de los estudiantes y egresados. El análisis, digital en porcentajes, de estos datos sirve conocer el nivel de lengua de arranque de la propuesta curricular. Se utilizó la prueba que aplica la ELCE para realizar exámenes de suficiencia de la lengua inglesa.

2. La encuesta: se realizó como técnica cuantitativa, se aplicó en el CURVA, bajo supervisión y orientación personalizada, a la población de 44 estudiantes y 15 egresados. Consiste en un cuestionario bastante amplio de 4 páginas. Se utilizó para obtener información diagnóstica sobre la necesidades subjetivas o de motivación, las necesidades objetivas-comunicativas lingüísticas, las necesidades de aprendizaje o estilos de aprendizaje y las necesidades socioprofesionales 0 interculturales que tienen los estudiantes y egresados de agroindustria en cuanto a competencias en la lengua inglesa. Se tabuló la información que luego fue analizada y sirvió de base para la propuesta curricular.

3. La entrevista semidirigida: se realizó, como técnica cualitativa, con los empleado- 
res que tienen o tendrán los egresados de la carrera. Se identificaron los empleadores más importantes a visitar en la región. Se sintetizó y tabuló, en la medida de lo posible, la cuantificación de la información, la cual se analizará para conformar la propuesta curricular.

4. El grupo focal: se realizó con 7 maestros y autoridades, en las instalaciones del CURVA; se usó como técnica cualitativa para determinar las necesidades de formación de los estudiantes de agroindustria, desde la visión de sus docentes y autoridades en cuanto a competencias en la lengua inglesa y de esta forma analizar técnicamente e identificar los lineamientos curriculares y pedagógicos que tendrá la propuesta curricular.

5. Análisis de documentos: se utilizó también esta técnica de investigación para la recolección de datos válidos. Se analizaron 2 documentos oficiales: el plan de estudios de la Carrera de Agroindustria con los contenidos sintéticos de todas las asignaturas del campo disciplinar propiamente, facilitado por la coordinación de dicha carrera. También se examinó el documento de diagnóstico de necesidades llevado a cabo para la creación de esa carrera.

Es importante rescatar que el análisis de ambos documentos proporcionó valiosa información técnica o teórica sobre las competencias lingüísticas y socioculturales en la lengua inglesa que deben tener los estudiantes y egresados de agroindustria para garantizar un éxito académico y profesional, esto datos enriquecerán la propuesta curricular.

En resumen, el estudio se llevó a cabo en tres etapas: la diagnóstica, es decir, la aplicación de instrumentos para recogida de datos, tabulación y análisis de los mismos; la estructuración de la propuesta curricular a partir del análisis de los datos arrojados por las tres técnicas de investigación, la bibliografía y la literatura pertinente; y la revisión y validación, con guías de evaluación, por expertos identificados en la disciplina, con respecto a los indicadores de la validez, pertinencia, viabilidad, calidad y confiabilidad de la investigación y de la propuesta, previa su implementación.

\section{RESULTADOS Y ANÁLISIS}

A continuación se muestra una breve selección de los gráficos y análisis de los datos arrojados por los instrumentos de investigación: en primer lugar, un grupo focal con autoridades y docentes de la Carrera de Agroindustria del CURVA, luego se ven los resultados de la encuesta aplicada y discutida con los estudiantes y egresados; finalmente, se observan cualitativamente los datos obtenidos con la entrevista a los 
empleadores de los egresados del CURVA, así como la prueba de medición inicial del nivel de lengua inglesa.

\section{Gráfico 1. Disciplinas específicas profesionales en que se ocupa el inglés}

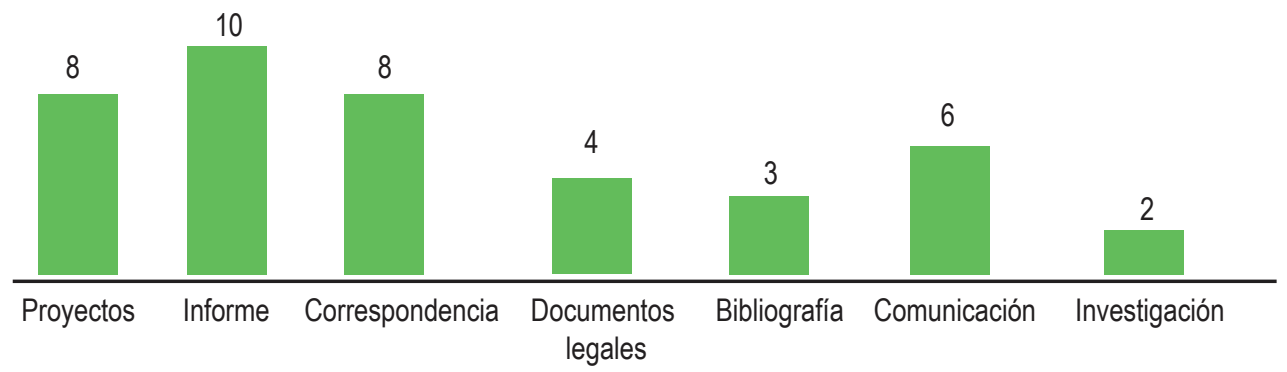

Fuente: Elaboración propia

Los docentes manifestaron que los estudiantes, principalmente egresados del CURVA, se enfrentan a las necesidades de leer y redactar informes, variada tipología de documentos y correspondencia en la lengua inglesa. Igualmente, deben hacer exposiciones orales de estos informes, así como proyectos en el campo de la agroindustria realizados o por contratar. La asistencia y participación en investigaciones científicas y de estudios de mercado es muy frecuente.

El programa de inglés tendrá que diseñar estrategias que permitan desarrollar competencias lingüísticas inter culturales en estas situaciones académicas, sociales y laborales.

\section{Gráfico 2. Cómo será útil el inglés en la carrera}

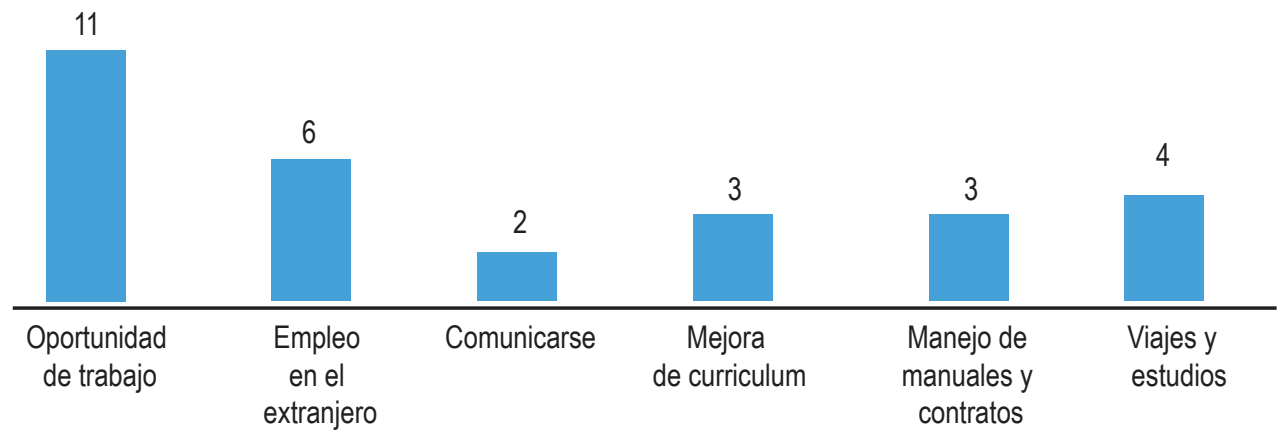

Fuente: Elaboración propia 
Los docentes manifestaron que los estudiantes, principalmente egresados del CURVA, se enfrentan a las necesidades de leer y redactar informes, variada tipología de documentos y correspondencia en la lengua inglesa. Igualmente, deben hacer exposiciones orales de estos informes, así como proyectos en el campo de la agroindustria realizados o por contratar. La asistencia y participación en investigaciones científicas y de estudios de mercado es muy frecuente.

El programa de inglés tendrá que diseñar estrategias que permitan desarrollar competencias lingüísticas inter culturales en estas situaciones académicas, sociales y laborales.

\section{Gráfico 3. Cómo está aprendiendo inglés}

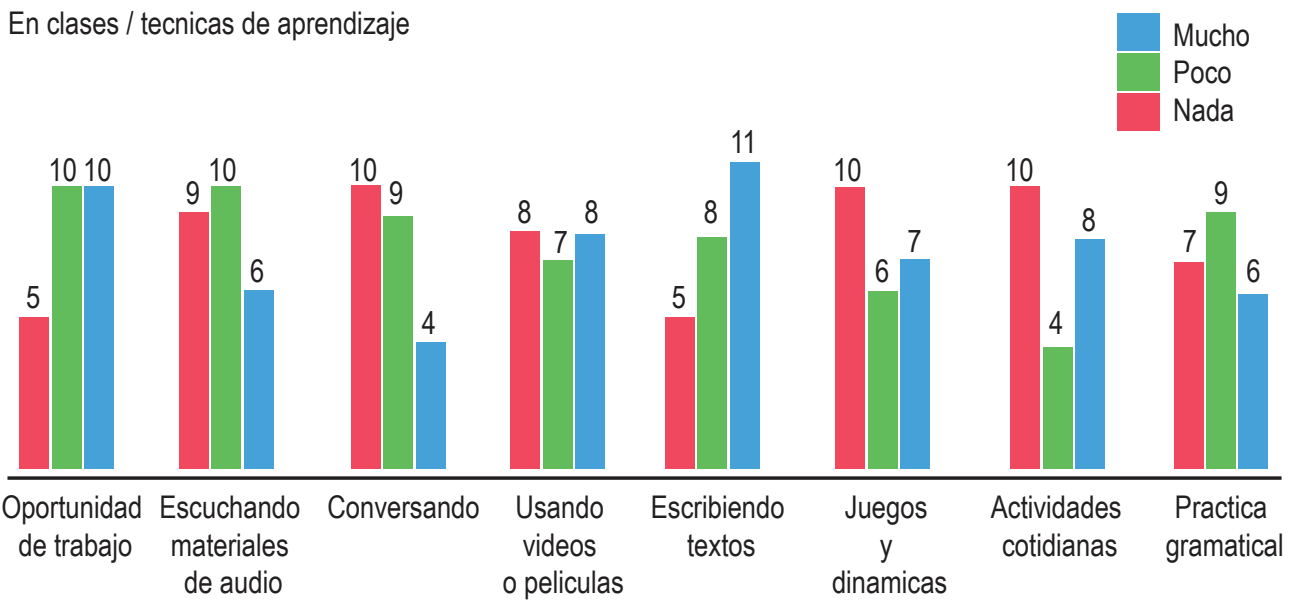

Fuente: Elaboración propia

Este gráfico 3 muestra cómo se mide la variable estilos de aprendizaje o buenas prácticas, ya ejercitadas por parte de los estudiantes en lo que respecta al aprendizaje de la lengua inglesa. La práctica más realizada por los estudiantes es la lectura y redacción de diferente tipos de textos. También mencionaron que es útil escuchar audios de diferentes medios, así como conversar sobre temas del trabajo, estudio y otras actividades cotidianas. Las dinámicas y juegos son, también, otras estrategias dentro y fuera de clase que el programa de inglés para el CURVA tendrá que considerar. 


\section{Gráfico 4. Medición del nivel de lengua en inglés}

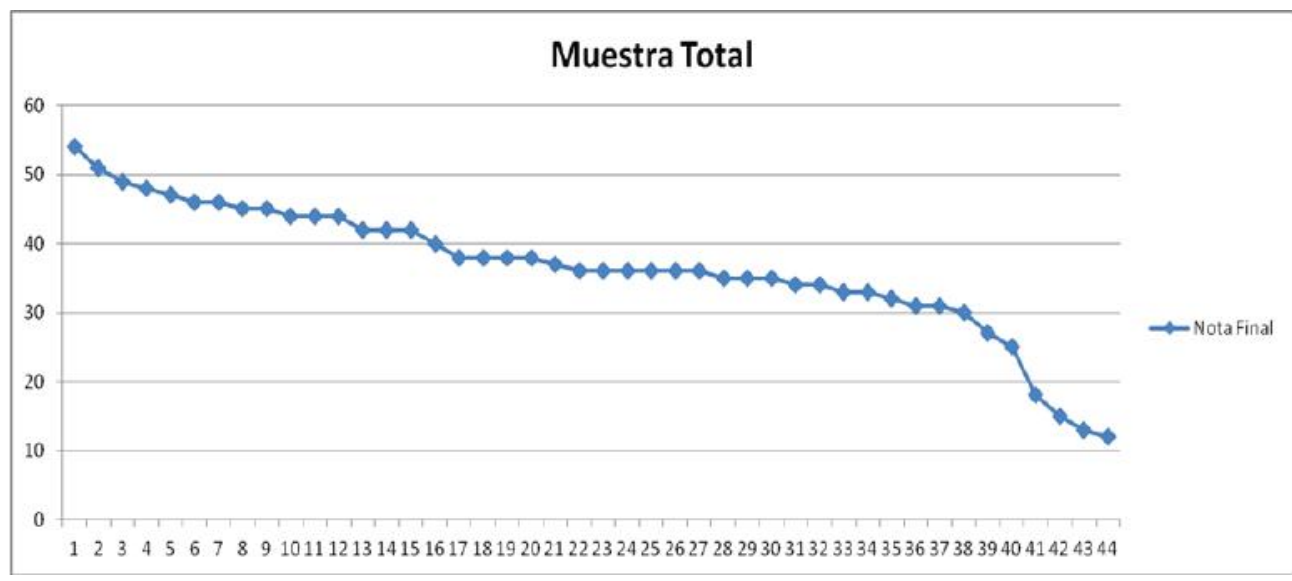

Fuente: Elaboración propia

El promedio, con base al $100 \%$, de notas de la muestra es de $36.55 \%$, la moda es de 36 y la mediana es de 36.261; el estudiante de máximo puntaje obtuvo un $51 \%$ y el menor fue de $11 \%$, aunque la mayoría obtuvo un resultado entre $40 \%$ y 30\%, razón por la cual se ubican, según el Marco Común Europeo de Referencia para las Lenguas (2005), en nivel A2.

Estos estudiantes se caracterizan por ser capaces de comprender frases y expresiones de uso frecuente relacionadas con áreas de experiencia que le son especialmente relevantes (información básica sobre sí mismo y su familia, compras, lugares de interés, ocupaciones, etc.). Saben comunicarse a la hora de llevar a cabo tareas simples y cotidianas que no requieran más que intercambios sencillos y directos de información sobre cuestiones que le son conocidas o habituales. Saben describir en términos sencillos aspectos de su pasado y su entorno, así como cuestiones relacionadas con sus necesidades inmediatas.

\section{CONCLUSIONES}

1. En primer lugar, se lograron identificar, desde 4 poblaciones diferentes, las necesidades de formación: en cuanto a las necesidades subjetivas o de motivación, se comprobó a través de las encuestas y entrevistas, así como con la prueba de medición, que el nivel de lengua de los estudiantes es básico e inicial en las todos 
los campos lingüísticos, hecho que motiva a los estudiantes hacia el logro de la meta: que es el aprendizaje apremiante, comprometido y autodeterminante de la lengua inglesa. Se consiguió determinar, además, cuales son las razones principales que motivan al estudiante para aprender inglés, entre las que se pueden resumir están: obtener mejores oportunidades de trabajo, mayor competitividad, más acceso a bibliografías y oportunidades de educación en el extranjero. En esta categoría también se identificaron cuáles son los estilos de aprendizaje que permiten una mejor adaptación al curso, tales como: trabajando en grupos y parejas, leyendo, escuchando y redactando textos auténticos del ámbito agroindustrial; conversando sobre actividades cotidianas, académicas y profesionales; realizando juegos y dinámicas; usando videos y otro material de audio, entre otras opciones; $y$, en menor medida, haciendo prácticas gramaticales. Igualmente se determinó que el estudiante necesita que el docente le presente ejemplos reales y de interés académico y vocacional.

En cuanto a las necesidades objetivas-comunicativas lingüísticas, se identificó que los estudiantes requieren un registro de lengua primeramente familiar, estándar, para luego pasar a un registro fuertemente intelectual, académico y vocacional. En esta categoría se identificó, también, que las competencias lingüísticas a desarrollar en los estudiantes, mediante la implementación de la propuesta curricular, tendrán un énfasis casi equitativo en lo que respecta a la comprensión y expresión escrita, como a la comprensión y expresión oral.

Finalmente, en cuanto a las necesidades socioprofesionales o interculturales, se identificó que la población estudiantil necesita desarrollar competencias de comprensión y expresión oral en inglés en actividades como, resumidamente, participar activamente en reuniones de trabajo, presentación de proyectos, investigaciones e informes, mantener diálogos en forma personal y por el teléfono; así como, entre otros, asistir a seminarios, talleres, conferencias nacionales y principalmente internacionales.

En lo que respecta a la comprensión y expresión escrita, en resumen, los estudiantes tienen la necesidad de leer y comprender más específicamente correspondencia en inglés en físico y virtual, contratos, ofertas y una amplia y variada tipología de textos escritos de índole académico y laboral. Necesitan también poder redactar textos variados como: hojas de vida, correspondencia en físico y digital, informes de estudios, proyectos, asignaciones especificas, procesos industriales y de mercadeo, polizas, reportes financieros, inventarios y otros.

2. A raíz de los resultados y a la luz del marco teórico, se logró diseñar y, posteriormente, validar técnicamente por expertos de ciudad universitaria y del CURVA, un programa de propuesta curricular de lengua inglesa por objetivos específicos intensivo, basándose en los datos obtenidos por los instrumentos de recolección de datos, para la Carrera de Agroindustria del CURVA en la UNAH que evidencia- 
rá y responderá eficazmente a las necesidades de formación de los estudiantes y egresados. La propuesta tendrá una duración de un semestre académico y de 14 a 15 horas de clases por semana, precedido por un programa obligatorio de inglés intensivo de formación general de un año de duración o de tres periodos académicos, lo cual garantizará el nivel intermedio de lengua inglesa para ingresar al programa de inglés con fines específicos para la Carrera de Agroindustria, se puede observar más claramente de forma gráfica esta malla curricular en la figura $1^{2}$.

\section{Figura 1. Esquema de la propuesta curricular}

\section{Propuesta programa de inglés con fines específicos para comercio internacional - UNAH}
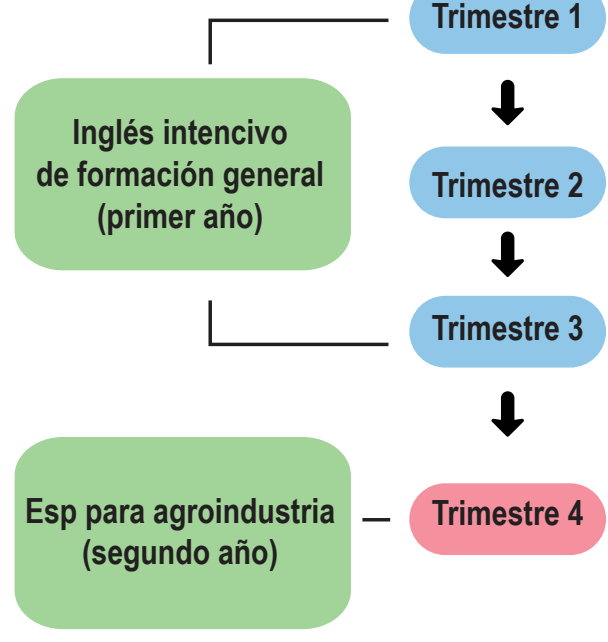

Opcional
Trimestre 1

- 2 horas diarias: lunes a jueves

-14 o 15 horas a la semana = trabajo

- virtual complentario - Bimodal

-516 hrs

- A1 + (B1) / MCERL / 4 Skills

- Introducción de contenidos de agroindustria
-Necesidades: son la base la propuesta curricular - Content Based / Intertransdiciplinariedad -Material: Auténtico, Selección/ diseño de texto -Desarrolo de competencias especificas en área de Agroindustria

- Reforzamiento de Nivel de Lengua: B2/ 7246 hrs 0 Niveles Bajos: CE---CO o Niveles Altos: EO--EE

\section{Curso de especialización intensiva}

\footnotetext{
${ }^{2}$ No es posible presentar en este artículo la propuesta curricular por su extensión, sin embargo, se
} podrá compartir con interesados mediante contacto a este correo: jose.espino@unah.edu.hn 


\section{BIBLIOGRAFÍA}

Cabré, M.T. (2010). Recursos lingüísticos en la enseñanza de lenguas de especialidad. Institut Universitari de Lingüística Aplicada. Universitat Pompeu Fabra (Barcelona). Recuperado de: http://cvc.cervantes.es/obref/aeter/conferencias/cabre.htm

Consejo Europeo. (2005). Marco Europeo Común de Referencias para las Lenguas: apprendre, enseigner, évaluer. Paris: Didier.

Mangiante, J. M. y Parpette, Chantal (2004). Le Français sur Objectifs Spécifiques: de l'analyse des besoins à l'élaboration d'un cours. Paris: Hachette FLE.

Mangiane, Jean Marc. (2007). Une démarche de référentialisation en français des professions: le partenariat universités - Chambre de Commerce et d'Industrie de Paris (CCIP). Université d'Artois. Recuperado de: http://www.fdlm.org/fle/ra/articles/modele.php

Porcher, L. (2004). L'Enseignement des Langues Étrangères. Paris: Hachette Éducation.

Vez, J.M. (2006). Formación en didáctica de las lenguas extranjeras. Santa Fe: Homo Sapiens. 